\title{
Micro-Autologous Fat Transplantation (MAFT) for Dorsal Hand Rejuvenation
}

\author{
EHAB Z. AZZAM, M.D.*; WALID M. ELMIDANY, M.S.** and AHMED A. ZIDAN, M.D.* \\ The Department of Plastic and Reconstructive Surgery, Main University Hospital, Alexandria University* and \\ The Department of Plastic and Reconstructive Surgery, School Children Hospital, Health Insurance Authority, Alexandria**, \\ Egypt
}

\begin{abstract}
Introduction: The dorsal surfaces of our hands are highly visible parts of the body. However with ageing, the aesthetic aspects of the hand deteriorate both in skin quality and contour with obvious appearance of dorsal blue veins and extensor tendons and loss or absence of subcutaneous fat tissue. The literature reports multiple options for addressing this problem, yet, all of these choices come with their own complications. Micro-autologous fat transplantation (MAFT) can be used in hand rejuvenation by a combined effect. First, it is possible to augment the dorsum of the hand and enabling visible blue and tortious veins as well as extensor tendons to be covered, resulting in a hand with uniform contour. Second, the fat cells have a beneficial effect on deep and superficial skin tissue via adipocytes derived stem cells (ASCs), thereby rejuvenating the hand. In this study, we performed (MAFT) technique to the three dorsal subcutaneous laminae for the rejuvenation of dorsal hand surfaces and determined its clinical results.
\end{abstract}

Patients and Methods: MAFT was performed in 10 female patients. Fat was harvested by performing liposuction from different body sites, then processed, and refined by decantation. Purified fat was micro-transplanted to the dorsal surface of the hand in parcels of small volume. Post treatment checkups were performed at $24 \mathrm{~h}, 7$ days, 1 month, 3 months, and 6 months after treatment to evaluate the healing cascade of the hand. Patient-rated satisfaction was reported during the patient's final visit (at least 6 months after MAFT) using a 5point scale. The Merz Hand Grading Scale (MHGS) was used to grade the appearance of the dorsal hand preoperatively and 6 months after MAFT.

Results: The mean age was 46 years (range, 36-60 years). The time taken for MAFT injection of both hands was in an average of $30 \mathrm{~min}$. On average, the fat volume delivered was 20 and $18.5 \mathrm{~mL}$ for the right and left hands, respectively. No major complications were reported, only mild to moderate swelling was noted, which subsided after 7 to 10 days postoperatively. Favorable outcomes were noted in patients which was reflected on the patient satisfaction rate (very satisfied, $60 \%$ and satisfied, $30 \%$ ) and the remarkable up grading in the Merz Hand Grading Scale.

Conclusion: In conclusion, the clinical results obtained using MAFT have demonstrated the feasibility and effectiveness of this approach in creating a younger and more beautiful dorsal hand appearance. However, longer studies in a larger patient population are required to assess long-term outcomes.

Key Words: MAFT - Dorsal hand - Rejuvenation.

\section{INTRODUCTION}

The dorsal surfaces of our hands are highly visible parts of the body. Studies have shown that people are able to roughly estimate a person's age solely by viewing their hands [1]. The dorsum of the hand typically has a smooth contour, without visible or with minimal dorsal veins and extensor tendons. However with ageing, the aesthetic aspects of the hand deteriorate including alterations of the skin quality with alteration of cutaneous pigmentation, the dorsal blue and tortious veins and extensor tendons become more obvious, and the contouring of the hands becomes uneven with loss or absence of subcutaneous fat [2].

The literature reports multiple options for addressing this problem, yet, all of these choices come with their own complications. Ablative procedures using tri-chloroacetic acid or phenol skin peels improve the quality of the skin by inducing dermal thickening and can also lighten pigmentation changes, however, Epidermis and basal membrane injury leading to fibrosis of papillary dermis and dys-pigmentation have been reported [3].

The application of some dermal filler materials as an aesthetic re-contouring and re-volumizing agents have been used [4], however, an increasing number of complications such as allergic reactions, filler material migration, injection necrosis, embolism and foreign body granuloma following their use as an have been reported, in addition, they dissipate with time and necessitate repetitive treatments $[\mathbf{5 , 6 ]}$. 
Surgical hand lift excises redundant skin and wrinkles from the dorsal hand, wrist, and forearm, however, Injury to superficial branch of radial nerve and dorsal branch of ulnar nerve, wound dehiscence, and decreased range of motion of wrist are not un common complications $[7,8]$.

Laser treatments such as Q-switched lasers, intense pulsed light, photodynamic therapy, ablative and non ablative fractionated lasers, radio frequency, and plasma skin regeneration provide a variety of visible effects including epidermal whitening, removal of visible lesions, improved skin texture, reduced noticeable wrinkling, and dermal remodelling, however, in addition to multiple treatment sessions, the risk of hyper-pigmentation, scarring, textural change, crusting and bleeding is still present $[2,9,10]$.

Fournier [11] in 1988 first described autologous fat grafting for hand rejuvenation by injecting a bolus of fat into an isolated area in the hand and subsequently massaging the injection over the entire surface. However, this technique had some drawbacks as regards contour irregularities and unpredictable fat survival. Later, Coleman [12] suggested blunt, subcutaneous tunneling with multiple injections and more consistent results.

Fat grafting has a combined effect in hand rejuvenation, By using autologous fat, it is possible to augment the dorsum of the hand, thus, allowing visible blue and tortious veins as well as extensor tendons to be covered, resulting in a hand with uniform contours. Furthermore, the fat cells have another beneficial effect on deep and superficial skin tissue via adipocytes stem cells, thereby rejuvenating the hand [13].

The dorsal hand anatomy relevant to hand rejuvenation has been previously described by Bidic et al. [14]. They discovered that the subcutaneous tissue in the dorsum of the hand is divided into three distinct fatty laminae with intervening fascia namely the dorsal deep lamina (DDL), dorsal intermediate lamina (DIL), and dorsal superficial lamina (DSL), which are partitioned by dorsal deep fascia (DDF), dorsal intermediate fascia (DIF), and dorsal superficial fascia (DSF) (Fig. 1). The dorsal veins and sensory nerves reside in the middle layer whereas the extensor tendons reside in the deepest one. The most superficial lamina contains no anatomic structures and is traversed with 8 to 12 septal adhesions, which contain perforating vessels supplying the subdermal plexus.

In this study, we performed the micro autologous fat transfer (MAFT) technique to the three dorsal subcutaneous laminae for rejuvenation of dorsal hand surfaces and determined its clinical results.

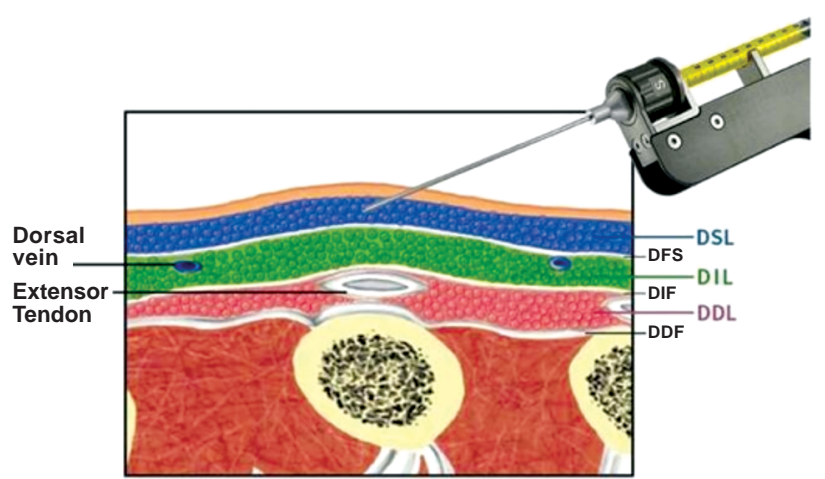

Fig. (1): The subcutaneous tissue of the dorsal hand is composed of three layers: Dorsal deep lamina (DDL), dorsal intermediate lamina (DIL), and dorsal superficial lamina (DSL), which are partitioned by dorsal deep fascia (DDF), dorsal intermediate fascia (DIF) and dorsal superficial fascia (DSF).

\section{PATIENTS AND METHODS}

Between June 2017 and December 2018, 10 female patients underwent liposuction from different body sites were simultaneously received MAFT for rejuvenation of the dorsal surfaces of their hands. The exclusion criteria included smoking, any history of trauma or filler injection for the dorsal hand surface, other co-morbidities including coagulation disorders, lipid metabolism disorders, severe chronic disease states and acute infections.

Patients were admitted mainly for lipoabdominoplasty in 4 cases, liposuction of the thighs in 3 cases and arm liposuction in 2 cases and only one case was seeking liposuction from her back rolls, thus, the abdomen, the thighs, the arms and the back were the main donor sites for harvesting our fat tissue.

\section{Surgical technique:}

Under general anaesthesia and with complete aseptic conditions, the donor site was infused with tumescent Klein's solution (1 liter of lactated Ringer's solution, $25 \mathrm{cc}$ of $2 \%$ lidocaine, and $1 \mathrm{cc}$ of 1:1000 epinephrine solution) to attain adequate skin turgor [15] After 10 to $15 \mathrm{~min}$, the fat was harvested through a 3-mm incisions made by 11 blade scalpel using a blunt tip suction cannula (diameter $3 \mathrm{~mm}$; with elliptical side sharp holes) connected to a $60 \mathrm{ml}$ syringe (Fig. 2A,B). Breaking fat with cannula before suction application made easier suction and allowed for gaining smaller particles of fat. The lipo-aspirate was then decanted for 10min; contaminants such as blood, serum, and oil were removed. After which, the purified fat 
was transferred in to $10 \mathrm{ml}$ syringes (Fig. 2C). Slight mechanical emulsification was then done by shifting the fat between two 10-cc syringes connected to each other by a 3 way adapter (Fig. 2D). After 2-3 passes, the micro fat was carefully transferred into a $1 \mathrm{cc}$ luer-slip syringe and was now ready for use (Fig. 3A,B).

The hands were prepared and draped in a sterile fashion. The area of the dorsum of the hand where fat graft will be injected must first be defined. The metacarpal bones were outlined longitudinally on the dorsum of the hand. Prominent dorsal veins were also identified and marked to reduce the risk of dorsal vein perforation (Fig. 3C).

The main stab incision was made at the dorsum of the wrist, but if needed, additional incisions were made between the metacarpal phalangeal joints of fingers (Fig. 3D).

A16g blunt cannula was tilted, inserted at $45^{\circ}$ down to the deepest plane (the dorsal deep lamina), and advanced distally towards the proximal interphalengeal joint. The cannula was then withdrawn while small fat parcels were injected as linear threads in a serial fanning distribution [9]. At the same entry points, the skin was tented up to open the dorsal superficial lamina space so that the cannula reliably enters that plane and again fat was injected in a retro grade fashion. The process was repeated in the the dorsal intermediate laminae with a great care not to injure or inject the dorsal veins. Approximately 10 to $15 \mathrm{~mL}$ of harvested fat was injected in the dorsum of each hand (in the three lamiae) (Fig. 1) and was sufficient to give it a slightly "puffy" look, in addition, 2 to $3 \mathrm{~mL}$ was injected in the snuffbox, and a smaller volume (1 to $2 \mathrm{~mL}$ ) in each dorsal webspace and each finger base [16,17] (Fig. 4). Once injected, the fat was gentelly massaged into place to create an even distribution and to obtain a smooth contour.

Incisions were closed with non- absorbable 5/0 sutures. The hands were then padded with an antibiotic ointment and gauze dressings, wrapped with an elastic bandage and were elevated for the next 48 hours. All Patients were warned that oedema and ecchymosis will be apparent for 1 to 2 weeks. Systemic antibiotics were prescribed for 10 days, beginning the evening before the surgery and nonsteroidal anti-inflammatory when needed.

Post treatment check-ups were performed at 24h, 7 days, 1 month, 3 months, and 6 months after treatment to evaluate the healing cascade of the hand. Standardized digital coloured photos were taken at each visit and compared pre- and postoperatively. In addition, early complications (cellulitis, digital numbness, infections) as well as late complications (cyst formation, fat necrosis, nodulations and reabsorption of the grafted fat) were meticulously recorded.

Patient-rated satisfaction was reported during the patient's final visit (at least 6 months after MAFT) using a 5-point scale. The Merz Hand Grading Scale (MHGS) [18] was used to grade the appearance of the dorsal hand preoperatively and 6 months after MAFT (Table 1).
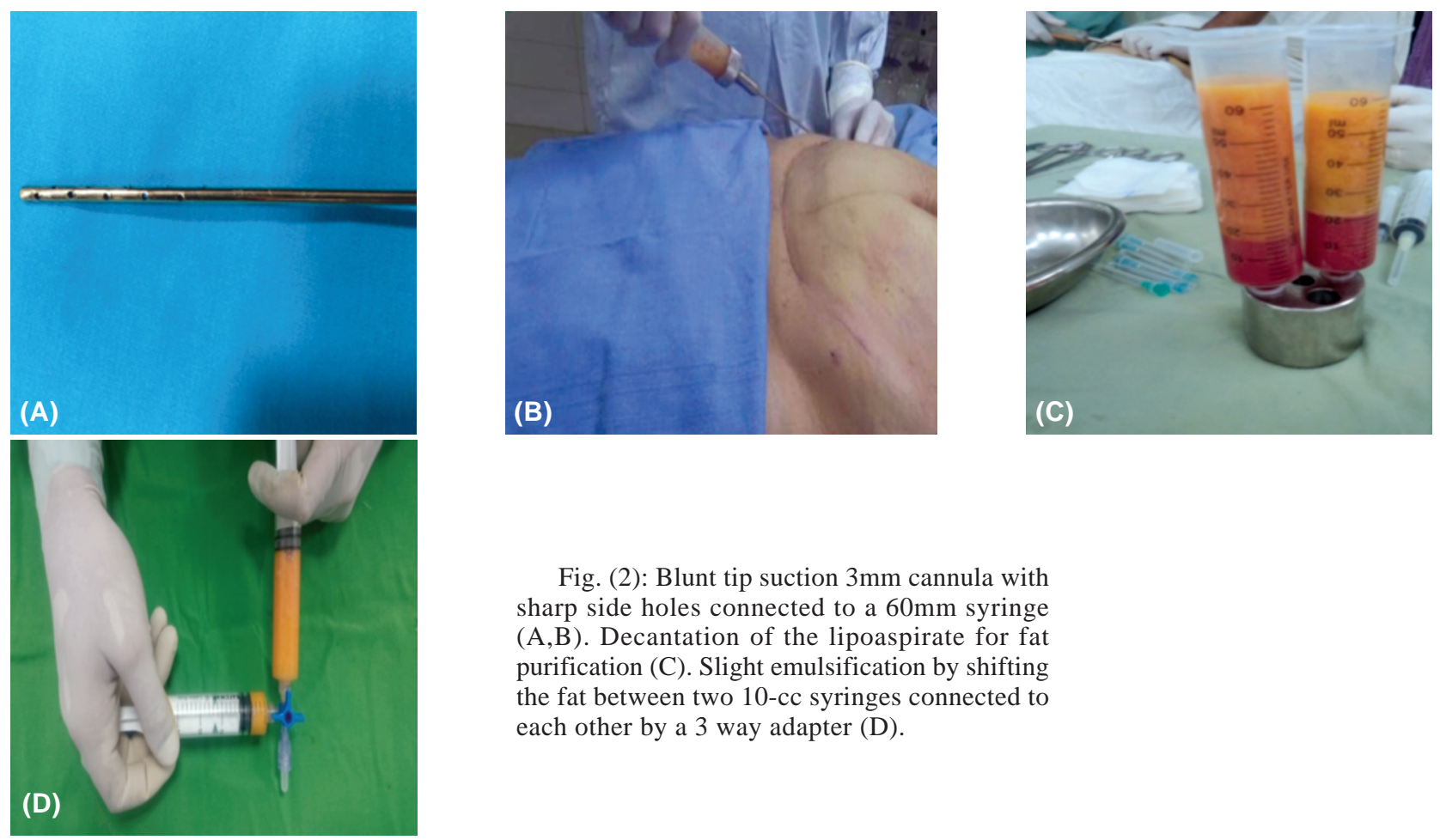

Fig. (2): Blunt tip suction 3mm cannula with sharp side holes connected to a $60 \mathrm{~mm}$ syringe $(A, B)$. Decantation of the lipoaspirate for fat purification (C). Slight emulsification by shifting the fat between two 10-cc syringes connected to each other by a 3 way adapter (D). 

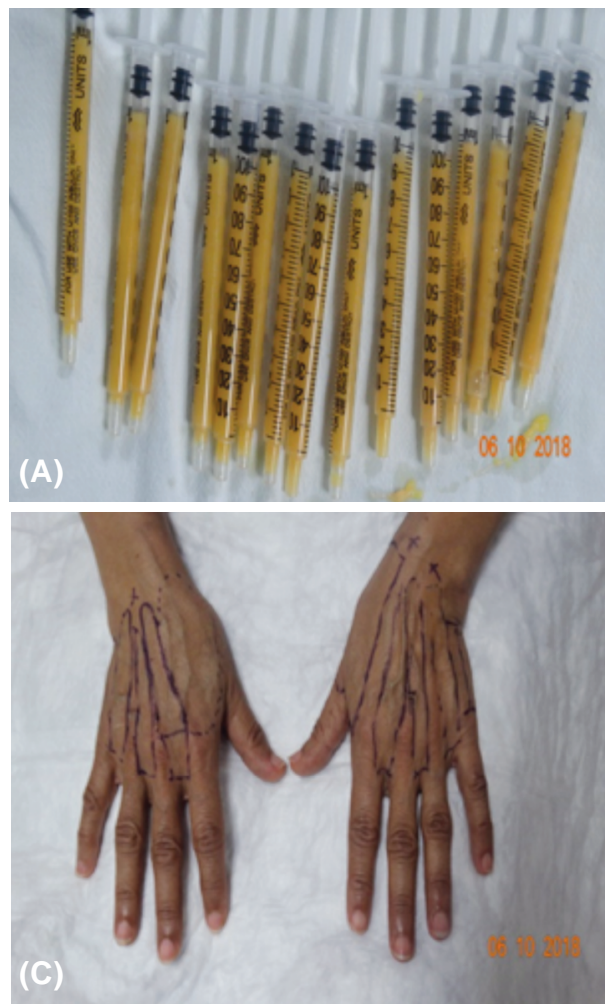
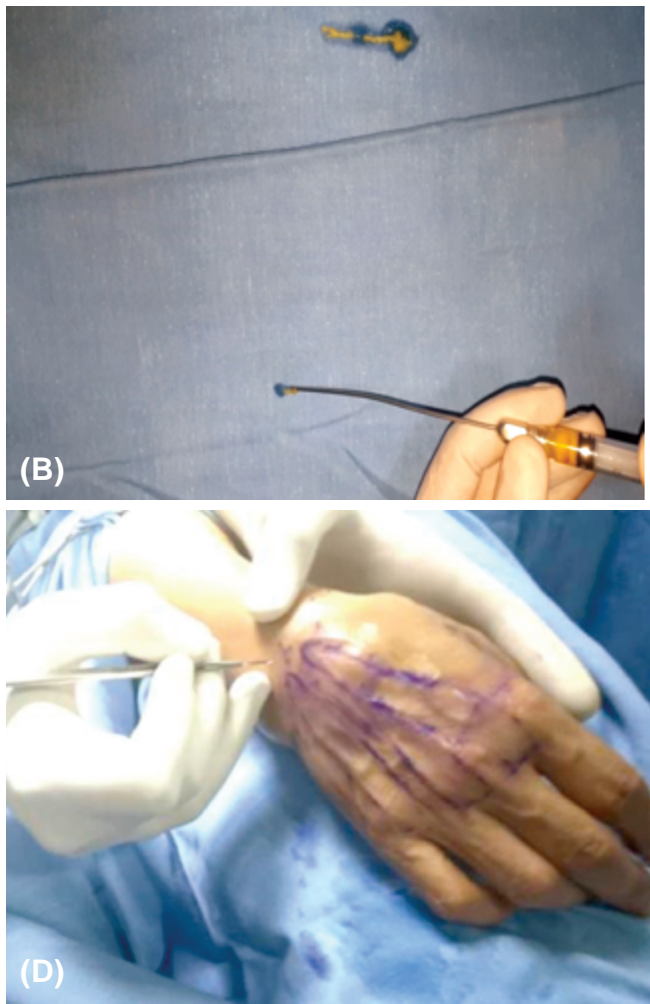

Fig. (3): Micro fat was carefully transferred into a 1cc luer-slip syringe and now ready for use (A,B). Identification and marking of prominent vasculature and the areas to be injected $(\mathrm{C})$. Stab incision at the main point of entry at the dorsum of the wrist (D).
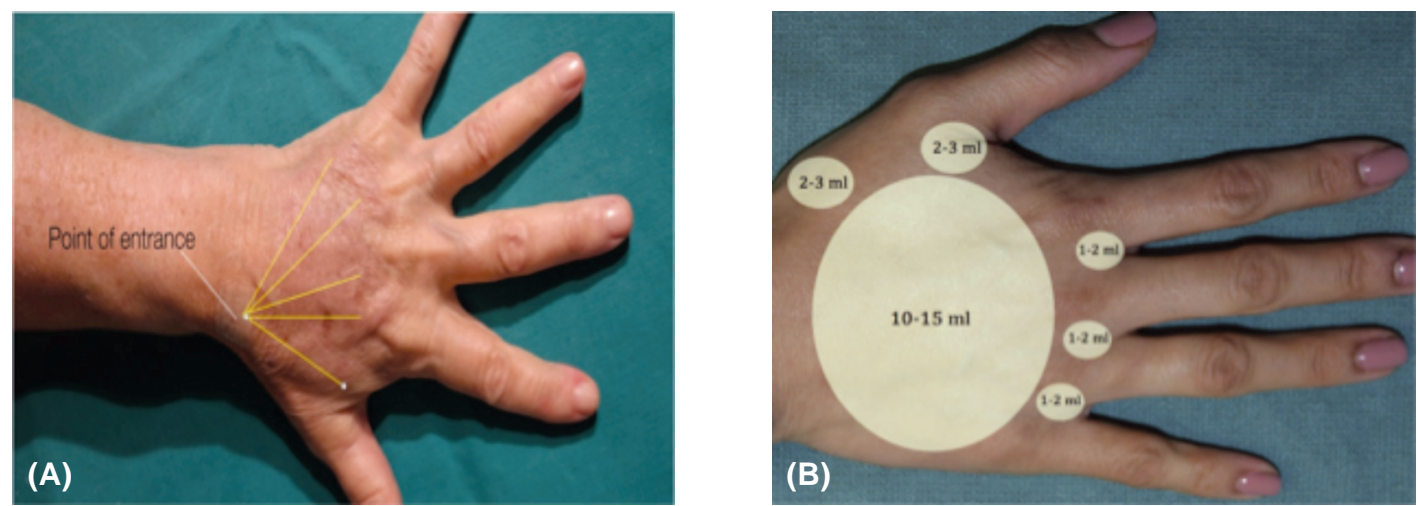

Fig. (4): Point of entry at the dorsum of the wrist (A). Volumes of micro fat grafting to the dorsum of the hand, ( 10 to $15 \mathrm{~mL}$ in the dorsum of the hand, 2 to $3 \mathrm{~mL}$ in the snuffbox, and 1 to $2 \mathrm{~mL}$ in each dorsal webspace).

Table (1): Merz hand grading scale.

\begin{tabular}{ccccc}
\hline 0 & 1 & 2 & 3 & 4 \\
\hline $\begin{array}{c}\text { No loss of } \\
\text { fatty } \\
\text { tissue }\end{array}$ & Mild loss of fatty tissue & $\begin{array}{c}\text { Moderate loss of } \\
\text { fatty tissue } \\
\text { Slight visibility of veins } \\
\text { Mild visibility of } \\
\text { veins and tendons }\end{array}$ & $\begin{array}{c}\text { Moderate visibility } \\
\text { of veins and tendons }\end{array}$ & $\begin{array}{c}\text { Marked visibility of } \\
\text { veins and tendons }\end{array}$ \\
\hline
\end{tabular}

\section{RESULTS}

The mean age was 46 years (range, $36-60$ years). The time taken for MAFT injection of both hands was in an average of $30 \mathrm{~min}$. On average, the fat volume delivered was 20 and $18.5 \mathrm{~mL}$ for the right and left hands, respectively. All patients were monitored for an average of 6 months (range, 48 months). No major complications (e.g., infection, neurovascular injury, cyst/nodulation formation, or uneven appearance) were recorded. Only mild to moderate swelling was noted, which subsided 
after 7 to 10 days postoperatively. The patientrated satisfaction scores obtained during the final visits are shown in Table (2).

As regards the MHGS, preoperatively, 20\% $(2 / 10)$ patients had grade $4,40 \%$ (4/10) patients had grade $3,30 \%(3 / 10)$ patients had grade 2 , and
$10 \%(1 / 10)$ patient had grade 1$)$ postoperatively, [20\% (2/10) patients had grade 2, 70\% (7/10) patients had grade 1 , and $10 \%(1 / 10)$ patients had grade 0] (Table 3).

Some clinical cases are illustrated in Figs. $(5,6,7)$.

Table (2): The patient-rated satisfaction scores.

\begin{tabular}{cccccc}
\hline $\mathrm{N}: 10$ & Very unsatisfied $(\%)$ & Un satisfisd $(\%)$ & Neutral $(\%)$ & Satisfied $(\%)$ & Very satisfied $(\%)$ \\
\hline 0 & 0 & $1(10)$ & $3(30)$ & $6(60)$ \\
\hline
\end{tabular}

Table (3): Pre- and post-MAFT comparison using of the (Merz Hand Grading Scale).

\begin{tabular}{|c|c|c|c|c|c|}
\hline $\mathrm{N}: 10$ & 0 & 1 & 2 & 3 & 4 \\
\hline & $\begin{array}{l}\text { No loss of } \\
\text { fatty tissue }\end{array}$ & $\begin{array}{l}\text { Mild loss of fatty } \\
\text { tissue }\end{array}$ & $\begin{array}{l}\text { Moderate loss of } \\
\text { fatty tissue }\end{array}$ & $\begin{array}{l}\text { Severe loss of } \\
\text { fatty tissue }\end{array}$ & $\begin{array}{l}\text { Very severe loss of } \\
\text { fatty tissue }\end{array}$ \\
\hline & & $\begin{array}{l}\text { Slight visibility of } \\
\text { veins }\end{array}$ & $\begin{array}{l}\text { Mild visibility of } \\
\text { veins and tendons }\end{array}$ & $\begin{array}{l}\text { Moderate visibility } \\
\text { of veins and } \\
\text { tendons }\end{array}$ & $\begin{array}{l}\text { Marked visibility } \\
\text { of veins and } \\
\text { tendons }\end{array}$ \\
\hline Pre-MAFT, $\mathrm{n}=10$ & 0 & $1(10 \%)$ & $3(30 \%)$ & $4(40 \%)$ & $2(20 \%)$ \\
\hline Post-MAFT, $\mathrm{n}=10$ & $1(10 \%)$ & $6(60 \%)$ & $2(20 \%)$ & $1(10 \%)$ & 0 \\
\hline
\end{tabular}
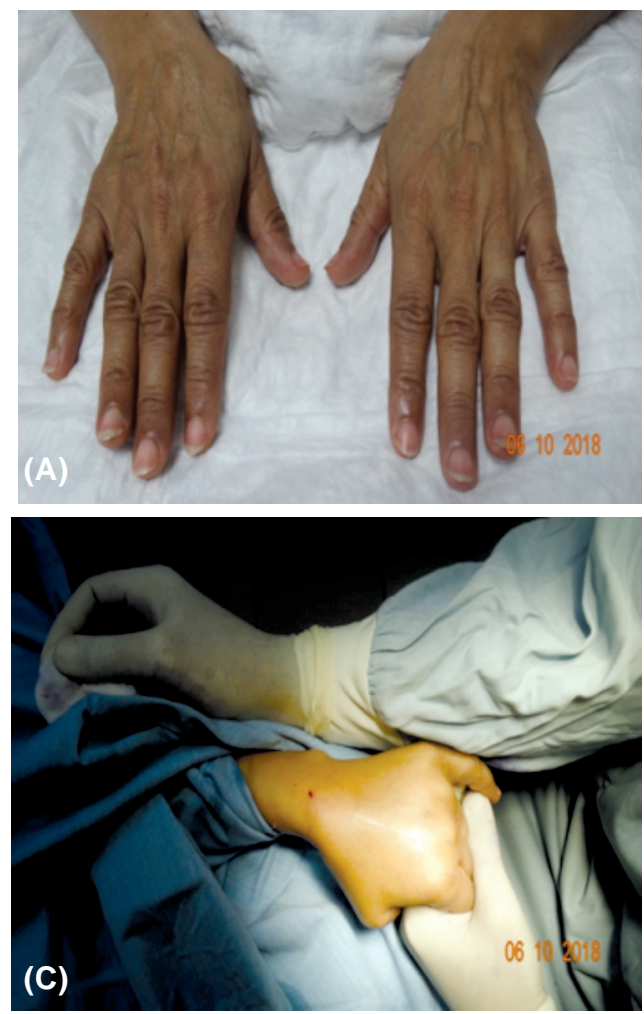
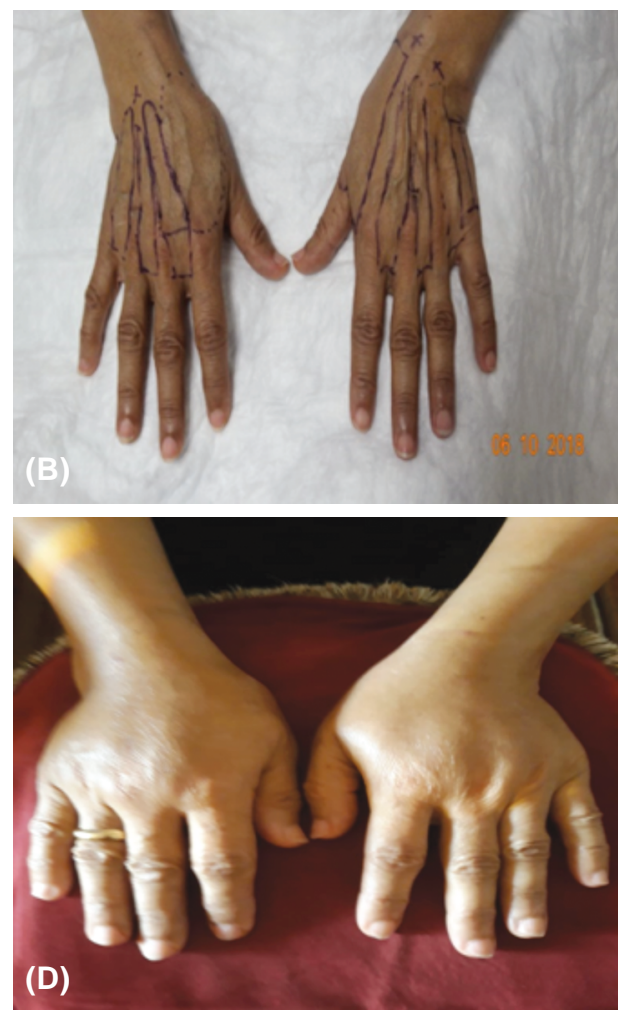

Fig. (5): (Case one). A 55 year old woman presented for lipo-abdominoplasty and fat grafting to restore the surface of her dorsal hands (A). Identification and marking of prominent vasculature and the areas to be injected (B). MAFT was performed to deliver 19 and $21 \mathrm{~mL}$ fat graft in her left and right hand respectively (C). Six months after the MAFT session, the fullness and restoration of both dorsal hands were maintained. The appearance of both hands was changed, from very severe loss of fatty tissue and marked visibility of veins and tendons (MHGS, grade 4) to mild loss of fatty tissue and slight visibility of veins (MHGS, grade 1). 

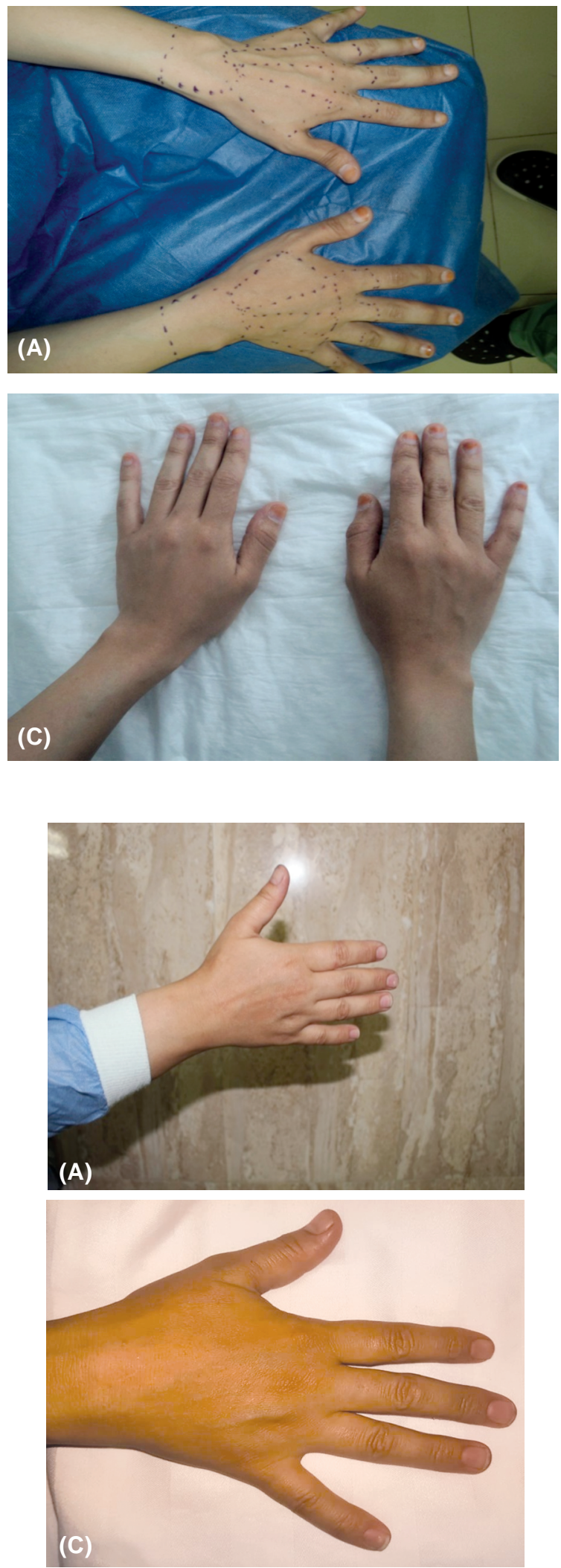

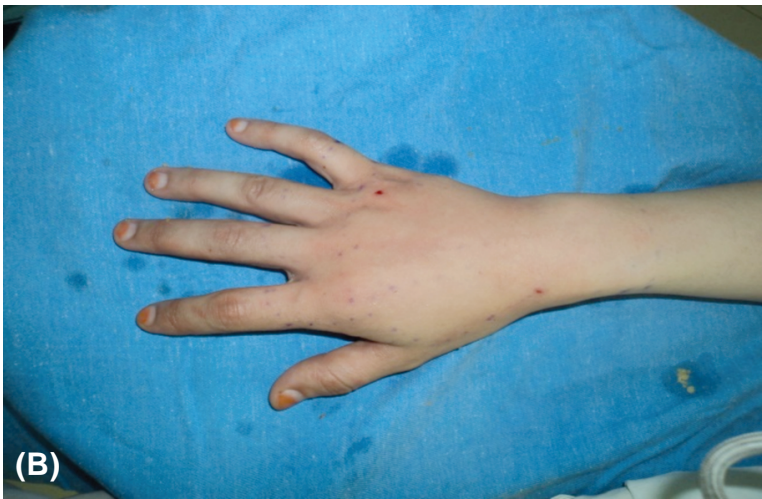

Fig. (6): (Case two) A 40 year old woman presented for liposuction of her back rolls and fat grafting to restore her dorsal hands. Identification and marking of prominent vasculature and the areas to be injected (A). MAFT was performed to deliver 18 and $16 \mathrm{~mL}$ fat graft in her left and right hand respectively (B). Six months after the MAFT session, the fullness and restoration of both dorsal hands were maintained. The appearance of both hands was changed, from moderate loss of fatty tissue with mild visibility of veins and tendons (MHGS, grade 2) to mild loss of fatty tissue and slight visibility of veins (MHGS, grade 1) (C).
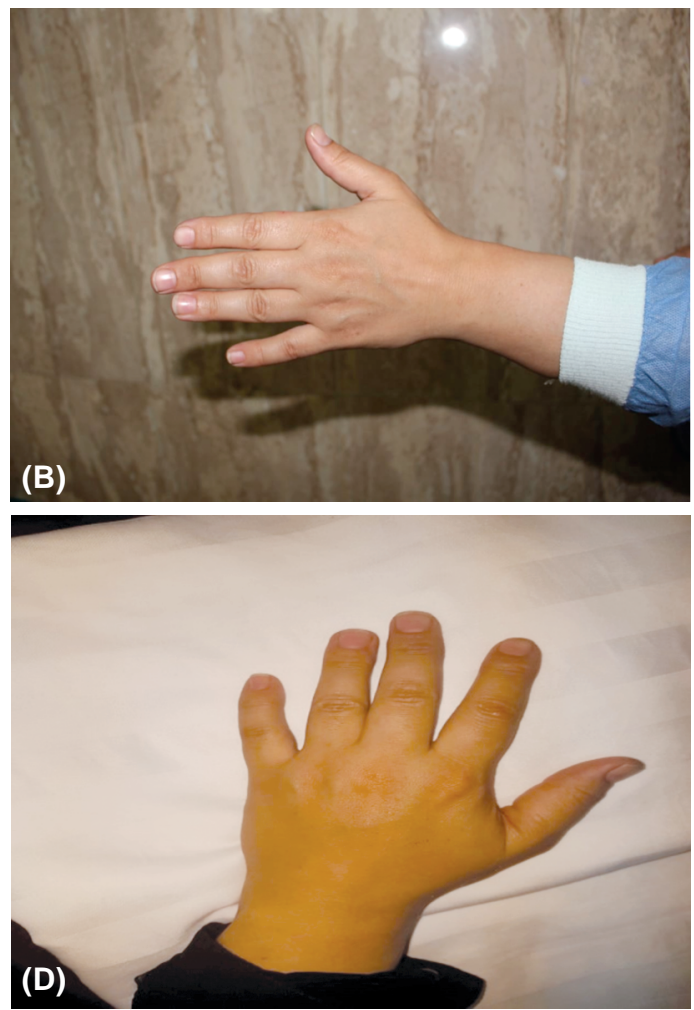

Fig. (7): (Case three): A 38 year old woman presented for lipo-abdominoplasty and fat grafting to restore the surface of her dorsal hands ( A,B). Six months after MAFT was performed to deliver 17.5 and $19 \mathrm{~mL}$ fat graft in her left and right hand respectively, the fullness and restoration of both dorsal hands were maintained. The appearance of both hands was changed, from moderate loss of fatty tissue and mild visibility of veins and tendons (MHGS, grade 2) to no loss of fatty tissue, no visible tendons and slight visibility of one vein (MHGS, grade 0). 


\section{DISCUSSION}

The appearance of our hands changes significantly during life. Recent studies [19] have confirmed that patients are concerned not only about hand function but also about hand appearance, particularly when it comes to reversing the effects of aging by restoring smooth contours and fullness.

Hand aging has been characterized by both extrinsic (environmental epidermal and dermal changes) and intrinsic (loss of subcutaneous fat, which reveals the underlying dorsal veins, extensor tendons, metacarpals, and metacarpophalangeal joints) factors [20]. Environmental related extrinsic factors can be improved with laser therapy, topical acids, bleaching agents, dermabrasion, and chemical peels [2], but age-related intrinsic changes are addressed primarily with restoration of subcutaneous volume.

Various synthetic fillers have been described for volume augmentation, however, many studies have reported adverse reactions when using synthetic fillers [21]. Autologous Fat cells represent biologic filler that can be safely used for tissue filling without fear of any allergic reactions, in addition, fat remains the treatment of choice because of the durability, volumization, and possible dermal regeneration [22].

The use of autologous fat for hand rejuvenation was first described by Fournier in 1988 [11], using a single large bolus that was then massaged over the dorsal surface. Years later, Coleman [12] modified his facial fat grafting technique and emphasized blunt tunnelling using smaller boluses which he believed increased smoothness and decreased fat resorbtion.

Sommer and Sattler [23] reviewed various approaches and success rates and concluded that good results have been reported regardless of technique as long as small volumes are used. Carpeneda [24] was the first of many to note that in histologic study, the diameter of a parcel of injected fat must be $3 \mathrm{~mm}$ or less for neovascularization to occur and for achieving favorable outcomes. In addition, the the parcel volume is critical to avoid occasional dislodgement of larger parcels, which in turn could result in nodulation and skin irregularity after fat grafting [25].

In this study, we placed micro autologous fat for volume restoration and rejuvenation of the dorsal surface of the hand in 10 female patients under general anaesthesia in the same setting of other plastic surgery operations. The human dorsal hand surface is composed of three anatomical layers namely DDL, DIL, and DSL.

In this study, we transferred an average of 20 $\mathrm{cc}$ and $18.5 \mathrm{cc}$ of micro autologous fat to the right and left hand respectively to the three anatomical layers (Fig. 1). Using blunt dissection and only one or two access points, as described in this technique especially during injection of the dorsal superficial lamina preserved the dorsal vessels and decreased the risk of their penetration; Furthermore, intravascular injection, is precluded by this MAFT technique because, first, the injection tip cannula was blunt and is therefore safer for the vessels. Second, the injection cannula size was $16 \mathrm{G}$, which corresponds to a diameter of $1.6 \mathrm{~mm}$. Anatomically, the dorsal veins of the hand have diameters around $1.27 \pm 0.48 \mathrm{~mm}$ [26]. Theoretically, the $16 \mathrm{G}$ injection cannula would not easily induce intraluminal penetration. Third, the extrusion pressure was also relatively low. Hence, the possibility of an intravenous administration with high retrograde flow pressure was almost completely eradicated. This was reflected in the decreased risk of haematoma and allowed for rapid resolution of ecchymosis and oedema in our cases. Nevertheless, a great caution was taken in all cases during MAFT to avoid any unintentional vascular insult. All our patients received general anaesthesia as the procedure was done in the same setting of other operations, we think that, meticulous performance with a steady appropriate anaesthesia were crucial during the procedure.

Coleman [22], stressed the importance of removing nonviable fat aspirated component such as oil, blood and lidocaine by centrifugation, however, Khater et al. [27] in a clinical and experimental study, presented their experience with different techniques of fat-processing. The authors concluded that in non-centrifuged adipose tissue, more activity preadipocytes are found, which could, which could possibly lead to enhanced changes of survival and even development of de novo fat. Another study compared the fat maintaence with centrifuge versus filtered and washed fat and concluded that the two fat-processing methods considered in this study yield results with similar quality [28]. In the present study, we used decantation process for the aspirated component to purify fat.

Favorable outcomes were noted in patients which was reflected on the patient satisfaction rate (very satisfied, $60 \%$ and satisfied, $30 \%$ ) and the remarkable up grading in the Merz Hand Grading Scale after only one session of MAFT. The advan- 
tages of MAFT in the clinical improvement include not only volume restoration of dorsal hand surfaces but also skin texture improvement. Adipocyte derived Stem cells (ASCs) may provide an explanation for the apparent healing effects seen with fat grafting.

Several articles have stated that (ASCs) are greatest in subcutaneous depots compared with visceral fat, with the highest concentrations in arm adipose tissue depots, and that the greatest plasticity is in ASCs isolated from inguinal adipose tissue depots [29]. However, we do not have a great deal of insight into the mechanisms of these effects. Some of the studies point to interaction that the grafted fat is having an effect on neighboring tissues either directly or through angiogenesis or vasculogenesis [30]. Other studies emphasize the plasticity between pre-adipocytes and macrophages, so that some or all of the healing effect may be secondary to enhanced immune response or removal of dying or defective cells leading to permanent tissue remodeling. Other factors may potentially be involved, such as the release of hormones, cytokines, or a growth factor [31].

In the literature, with use of the most up-todate techniques, autologous fat grafting is associated with few reported complications, the most common of them is reabsorption of the grafted fat [32]. In the current study, no patient required a second injection, this may be attributed to the relatively short follow-up period (average 6 months) and that some patients may require reinjection to achieve long-lasting results.

\section{Conclusion:}

In conclusion, the clinical results obtained using MAFT have demonstrated the feasibility and effectiveness of this approach in creating a younger and more beautiful dorsal hand appearance. In addition, autologous fat is readily available, easily obtained, inexpensive, host-compatible, and can be harvested repeatedly. Moreover, the rejuvenating effect of (ASCs) could also improve the skin texture and eliminate aging-related changes. However, longer follow-up period studies in a larger patient population are required to assess long-term outcomes.

\section{REFERENCES}

1- Bains R.D., Thorpe H. and Southern S.: Hand aging: Patients' opinions. Plast. Reconstr. Surg. 117 (7): 221218, 2005.

2- Butterwick K.J.: Rejuvenation of the aging hand. Dermatol. Clin., 23: 515-27, 2005.
3- Teimourian B. and Adham M.: Rejuvenation of the hand: Fat injection combined with TCA peel. Aesthet. Surg. J., 20 (1): 70-71, 2000.

4- Kuhne U. and Imhof M.: Treatment of the ageing hand with dermal fillers. J. Cutan Aesthet. Surg., 5: 163-9, 2012.

5- Park T.H., Yeo K.K., Seo S.W., et al.: Clinical experience with complications of hand rejuvenation. J. Plast. Reconstr. Aesthet. Surg., 65: 1627-31, 2012.

6- Lee S.K., Kim S.M., Cho S.H., Lee J.D. and Kim H.S.: Adverse reactions to injectable soft tissue fillers: Memorable cases and their clinicopathological overview. J. Cosmet Laser Ther., 17: 102-8, 2015.

7- Wendt J.R.: Distal, dorsal superior extremity plasty. Plast. Reconstr. Surg., 106 (1): 210-13, 2000.

8- Handle M., Bonfatti-Ribeiro L.M., Barcaro-Machado B.H. and Pitanguy I.: Minimal-scar handlift: A new surgical approach. Aesthet. Surg. J., 31 (8): 953-62, 2011.

9- Fabi S.G. and Goldman M.P.: Hand rejuvenation: Our experience. Dermatol. Surg., 38 (7): 1112-27, 2012.

10- Alster T.S. and Konda S.: Plasma skin resurfacing for regeneration of neck, chest, and hands: Investigation of a novel device. Dermatol. Surg., 33 (11): 1315-21, 2007.

11- Fournier P.F.: Who should do syringe liposculpturing? J. Dermatol. Surg. Oncol., 14: 1055-56, 1988.

12- Coleman S.R.: Hand rejuvenation with structural fat grafting. Plast. Reconstr. Surg., 110 (7): 1731-44, 2001.

13- Villanueva N.L., Hill S.M., Small K.H. and Rohich R.J.: Technical refinements in autologous hand rejuvenation. Plast Reconstr. Surg., 136: 1175-79, 2015.

14- Bidic S.M., Hatef D.A. and Rohrich R.J.: Dorsal hand anatomy relevant to volumetric rejuvenation. Plast. Reconstr. Surg., 126: 163-168, 2010.

15- Justin B.: Outcome Analysis of Combined Lipoabdominoplasty versus Conventional Abdominoplasty. Plast. Reconstr. Surg., 121: 1821, 2008.

16- Bank J., Fuller S.M., Henry G.I. and Zachary L.S.: Fat grafting to the hand in patients with Raynaud phenomenon. Plast. Reconstr. Surg., 133 (5): 1109-18, 2014.

17- Fabio Fantozzi1 Hand Rejuvenation with Fat Grafting: A 12-year single-surgeon experience. Eur. J. Plast. Surg., 40: 457-464, 2017.

18- Cohen J.L., Carruthers A., Jones D.H., et al.: A randomized, blinded study to validate the merz hand grading scale for use in live assessments. Dermatol. Surg., 41 (1): 384-8, 2015.

19- Chung K.C., Kotsis S.V., Kim H.M., Burke F. and Wilgis E.F.: Reasons why rheumatoid arthritis patients seek surgical treatment for hand deformities. J. Hand Surg. Am., 31 (2): 289-94, 2006.

20- Jakubietz R.G., Jakubietz M.G., Kloss D. and Gruenert J.G.: Defining the basic aesthetics of the hand. Aesthetic Plast. Surg., 29: 546-51, 2005.

21- DeLorenzi C.: Complications of injectable fillers. Aesthet. Surg. J., 33 (4): 561-75, 2013.

22- Coleman S.R.: Structural fat grafting: More than a permanent filler. Plast. Reconstr. Surg., 118 (3): 108-20, 2006. 
23- Sommer B. and Sattler G.: Current concepts of fat graft survival: Histology of aspirated adipose tissue and review of the literature. Dermatol. Surg., 26 (12): 1159-66, 2000.

24- Carpaneda C.A. and Ribeiro M.T.: Percentage of graft viability versus injected volume in adipose autotransplants. Aesthetic Plast. Surg. 18 (1): 17-19 1994.

25- Chou C.K., Lin T.M. and Chou C.: Influential factors in autologous fat transplantation-focusing on the lumen size of injection needle and the injecting volume. J. IPRAS, 9: $25-7,2003$

26- Kiray A., Ergur I., Tayefi H., Bağriyanik H.A. and Bacakoğlu A.K.: Anatomical evaluation of the superficial veins of the upper extremity as graft donor source in microvascular reconstructions: A cadaveric study. Acta. Orthop. Traumatol. Turc., 47: 405-10, 2013.

27- Khater R., Atanassova P., Anastassov Y., Pellerin P. and Martinot-Duquennoy V.: Clinical and experimental study of autologous fat grafting after processing by centrifugation and serum lavage. Aesthetic Plast. Surg., 33: 37-43, 2009.
28- Ali Asilian, Amir Hossein Siadat and Razieh Iraji: Comparison of fat maintenance in the face with centrifuge versus filtered and washed fat. J. Res. Med. Sci., 19 (6): 556-61, 2014

29- Mizuno H., Tobita M. and Uysal A.C.: Concise review: Adipose-derived stem cells as a novel tool for future regenerative medicine. Stem Cells, 30 (5): 804-10, 2012.

30- Nakagami H., Maeda K., Morishita R., et al.: Novel autologous cell therapy in ischemic limb disease through growth factor secretion by cultured adipose tissue-derived stromal cells. Arterioscler. Thromb. Vasc. Biol., 25: 2542, 2005.

31- Cousin B., Munoz O., Andre M., et al.: A role for preadipocytes as macrophage-like cells. F.A.S.E.B. J., 13: 305, 1999.

32- Vara A.D., Miki R.A., Alfonso D.T. and Cardoso R.: Hand fat grafting complicated by abscess. Hand, 8 (3): 348-51, 2013. 